\title{
ARTICLE
}

Received 5 Mar 2012 | Accepted 9 May 2012 | Published 12 Jun 2012

DOI: $10.1038 /$ ncomms1897

\section{Using light transmission to watch hydrogen diffuse}

\author{
Gunnar K. Pálsson ${ }^{1}$, Andreas Bliersbach ${ }^{1}$, Max Wolff ${ }^{1}$, Atieh Zamani ${ }^{1}$ \& Björgvin Hjörvarsson
}

Because of its light weight and small size, hydrogen exhibits one of the fastest diffusion rates in solid materials, comparable to the diffusion rate of liquid water molecules at room temperature. The diffusion rate is determined by an intricate combination of quantum effects and dynamic interplay with the displacement of host atoms that is still only partially understood. Here we present direct observations of the spatial and temporal changes in the diffusion-induced concentration profiles in a vanadium single crystal and we show that the results represent the experimental counterpart of the full time and spatial solution of Fick's diffusion equation. We validate the approach by determining the diffusion rate of hydrogen in a single crystal vanadium (001) film, with net diffusion in the [110] direction.

\footnotetext{
${ }^{1}$ Department of Physics and Astronomy, Materials Physics Division, Uppsala University, Box 516, Uppsala SE-75120, Sweden. Correspondence and requests for materials should be addressed to B.H. (email: bjorgvin.hjorvarsson@physics.uu.se).
} 
$\mathrm{D}$ iffusive motion is one of the most intriguing and investigated phenomena in physics. Fick formulated his two laws of diffusion $^{1}$ to which Einstein provided a microscopic foundation by considering atoms and molecules undergoing Brownian motion ${ }^{2}$. The interstitial diffusion of hydrogen in metals can be described in an analogous way, in which the motion results from discrete jumps between interstitial sites. The jump- and diffusion-rates are, therefore, intrinsically linked. As a consequence, the diffusion of hydrogen in metals can be inferred from measurements of jump rates in thermodynamic equilibrium, using, for example, nuclear magnetic resonance or quasi-elastic incoherent neutron scattering ${ }^{3}$. Determination of non-equilibrium diffusion can be performed by mechanical relaxation methods, such as the Gorsky effect ${ }^{3}$. Although these techniques have been extremely useful for determining the rate of hydrogen diffusion, they suffer from a severe shortcoming: the results do not provide any information about the evolution of concentration profiles in the samples. This severely limits the possibilities of validating many of the assumptions used when modelling the diffusion of hydrogen in materials.

The use of optical techniques to determine position resolved migration of hydrogen was pioneered by den Broeder et al. ${ }^{4}$, utilizing the metal to insulator transition in yttrium hydrides ${ }^{5}$. They could infer the diffusivity of hydrogen by following the frontier of the hydride formation as a function of position and time, by using the large change in the optical transmission resulting from the metal-insulator transition. Later, the same research group developed an approach based on an yttrium coating, whereby the metal insulator transition was used as a marker for the integrated flux of hydrogen in an underlying polycrystalline vanadium film ${ }^{6}$. Both approaches allowed the determination of the speed of the diffusion front, while no information could be obtained about the underlying concentration profiles. Here we present a method that provides time-resolved determination of the diffusion-induced concentration profiles in any metal, that exhibits hydrogen-induced changes of the density of states in the conduction band. The results are compared with the solution of the diffusion equation, and we will show that these provide full information about the development of the hydrogen content in the sample.

\section{Results}

Concentration conversion and geometrical aspects. A schematic illustration of the sample geometry and the instrument is shown in Fig. 1. The sample is covered with an amorphous aluminum oxide layer, except for a small opening at one end of the sample. The oxide is catalytically inactive and acts as a dissociation and diffusion barrier ${ }^{7}$. At $t=t_{0}$, molecular hydrogen is introduced into the evacuated chamber, which results in a selective dissociation of hydrogen on the catalytically active palladium surface. Atomic hydrogen is thereby introduced at one end of the sample and starts migrating laterally through the vanadium film. Vanadium exhibits a much stronger affinity for hydrogen, as compared with palladium, whereby the concentration in palladium can be safely neglected ${ }^{3,8}$. Hydrogen causes changes in the electronic structure, giving rise to changes in the optical transmission, which can be used to determine the concentration ${ }^{9,10}$ (for further details see the Methods section). The optical transmission is monitored as a function of time using a charge-coupled device, with a lateral resolution of about $20 \mu \mathrm{m}$, whereby the concentration of hydrogen as a function of both position and time can be determined.

The orientation of the vanadium single crystal with respect to the substrate ${ }^{11}$ and the resulting diffusion direction is illustrated in Fig. 2. The net diffusion direction of the hydrogen is in the [110] direction of the vanadium crystal, because of the directional relation of the crystallographic orientation to the catalytically active strip at one end of the sample. The total sample area is $10 \mathrm{~mm} \times 10 \mathrm{~mm}$ and the area of the catalytically active layer is $\sim 3 \mathrm{~mm} \times 10 \mathrm{~mm}$. The aspect

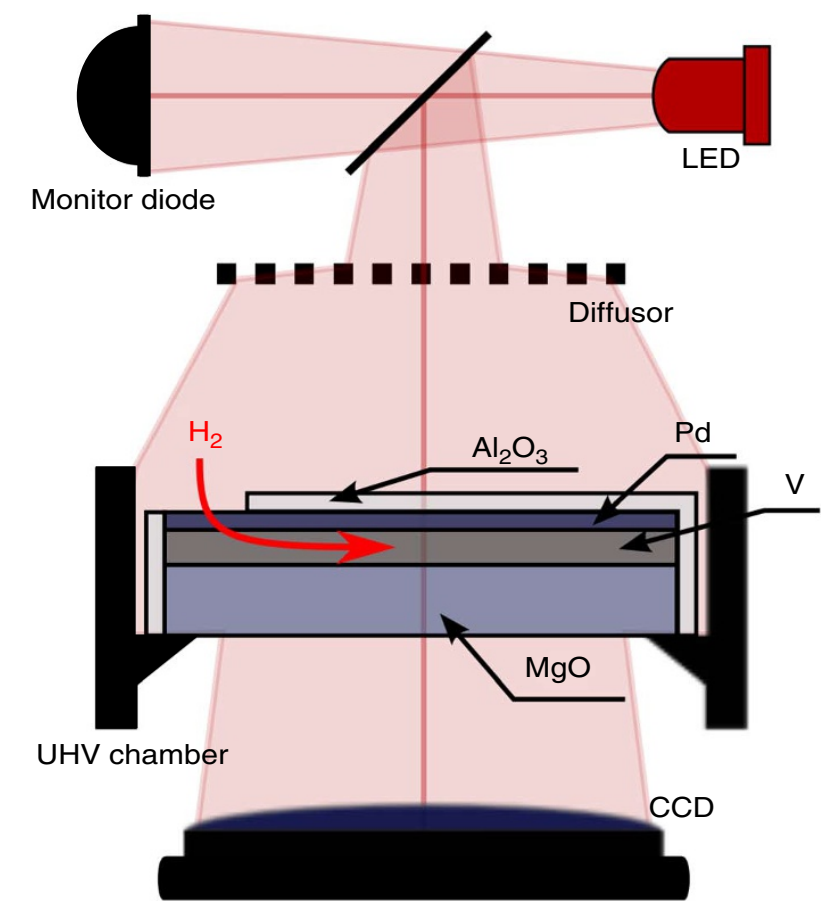

Figure 1 | Schematic illustration of the experimental approach. Monochromatic light, emitted by a light-emitting diode, shines on a semitransparent mirror, which is used to split the beam allowing simultaneous measurements of the transmission through the sample and the intensity of the source. A diffusor is used to homogenize the illumination, before the entry window of the ultrahigh vacuum chamber. The transmitted light is registered by a CCD camera. The sample is covered with $\mathrm{Al}_{2} \mathrm{O}_{3}$ to prevent hydrogen from entering and leaving, except for a small region at one end of the sample.

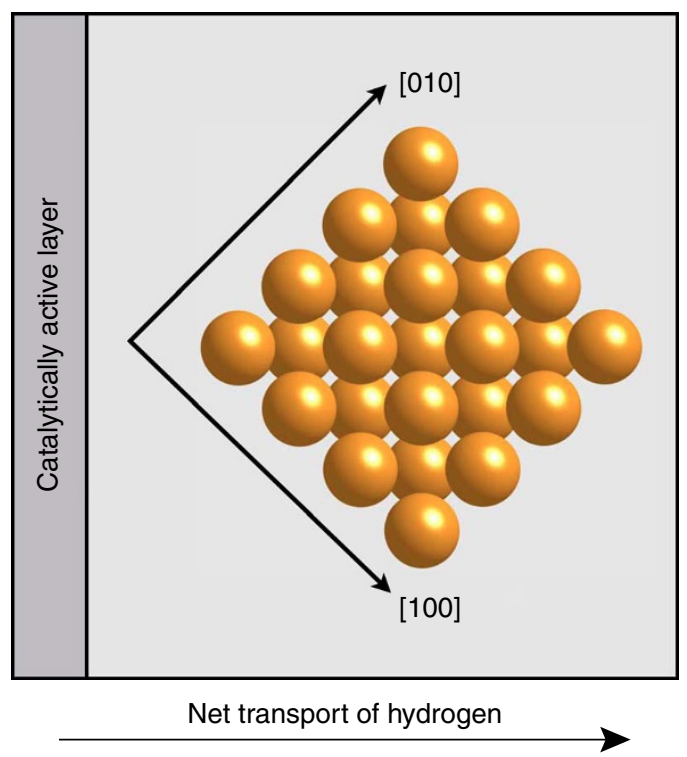

Figure 2 | Schematic top view of the sample and the orientation of the vanadium crystal. The epitaxial vanadium layer was grown on a $\mathrm{MgO}$ (001) substrate. The vanadium crystal is depicted by yellow spheres, and the in-plane lattice directions are indicated by arrows. The light grey area represents the oxide layer hindering the hydrogen uptake. The catalytically active stripe (dark grey) is parallel to one of the edges of the substrate, which results in a net diffusion of hydrogen in the [110] direction of the vanadium layer. 

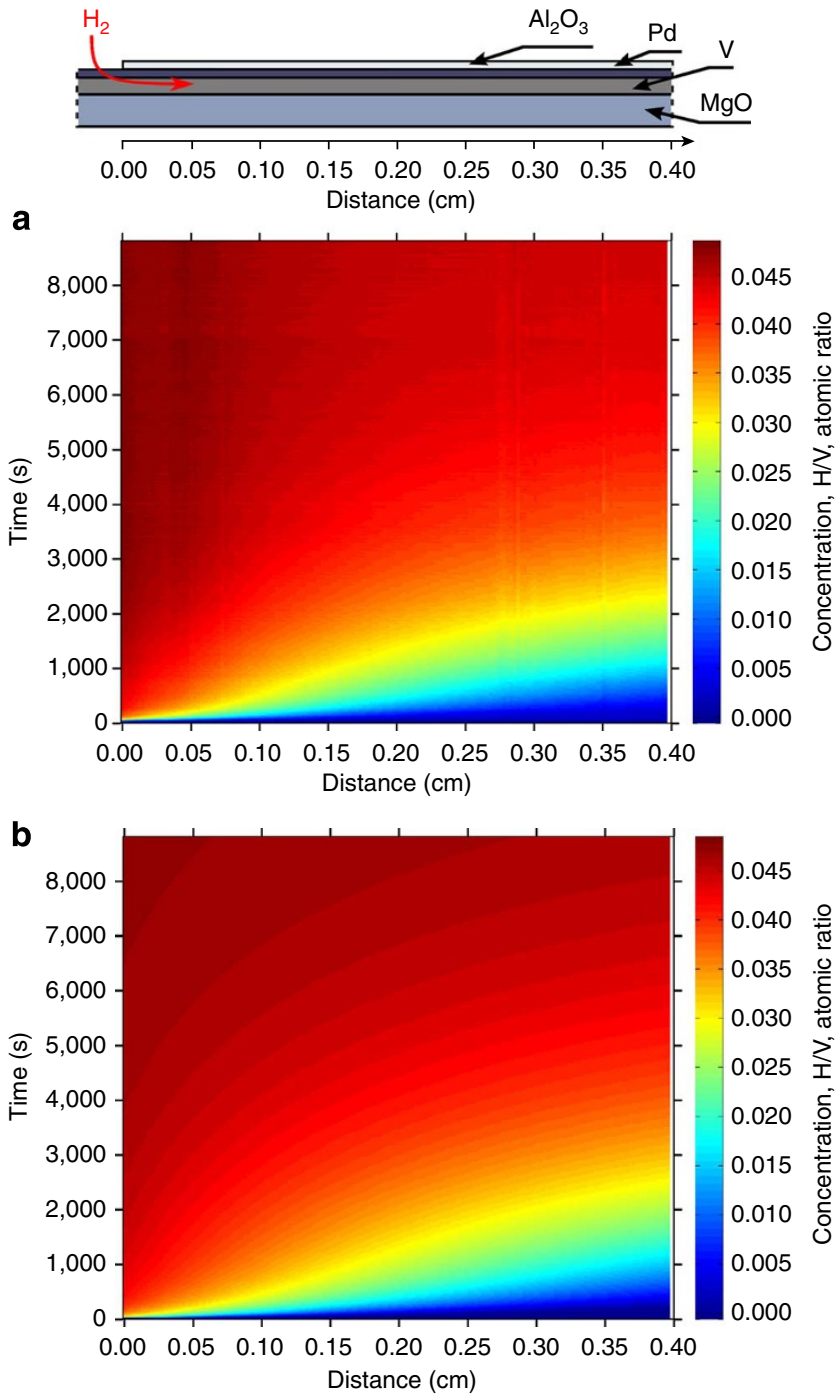

Figure 3 | Hydrogen concentration as a function of distance and time.

The experimental results are shown in (a) and the solution to Fick's second law of diffusion in (b). The measurements were performed at $493 \mathrm{~K}$ and with an external hydrogen pressure of $0.4 \mathrm{mbar}$. The insert at the top illustrates the sample geometry, with the end of the $\mathrm{Al}_{2} \mathrm{O}_{3}$ layer defining the starting point of the lateral diffusion.

ratio of the film thickness $(50 \mathrm{~nm})$ and the width of the stripe ensures equilibrated volume of hydrogen in the vanadium layer underneath the stripe that acts as a source or sink for the hydrogen diffusion.

Concentration profiles. By recording the concentration profile at short-time intervals, we can follow their temporal evolution and in Fig. 3a we display the results obtained at $493 \mathrm{~K}$. The horizontal axis represents the distance from the boundary where the hydrogen enters the sample, whereas the vertical axis specifies the time from the introduction of hydrogen in the chamber. The colour coding gives the concentration at any position and time during the measurements. The diffusion constant was determined from fitting the model described in equation (8) (see Methods section) to the data, using least squares minimization. The result is $D=5(2) \times 10^{-5} \mathrm{~cm}^{2} \mathrm{~s}^{-1}$ using line fits and $D=8(1) \times 10^{-5} \mathrm{~cm}^{2} \mathrm{~s}^{-1}$ using the whole dataset for $c=0.045 \mathrm{H} / \mathrm{V}$ (atomic ratio). This value can be compared with chemical diffusion constants obtained from Gorsky experiments ${ }^{12}$ that are 5.3(8) $\times 10^{-5} \mathrm{~cm}^{2} \mathrm{~s}^{-1}$ for $c=0.11 \mathrm{H} / \mathrm{V}$ and $9(2) \times 10^{-5} \mathrm{~cm}^{2} \mathrm{~s}^{-1}$ for $c=0.02 \mathrm{H} / \mathrm{V}$, respectively. In Fig. 3b, we display the calculated
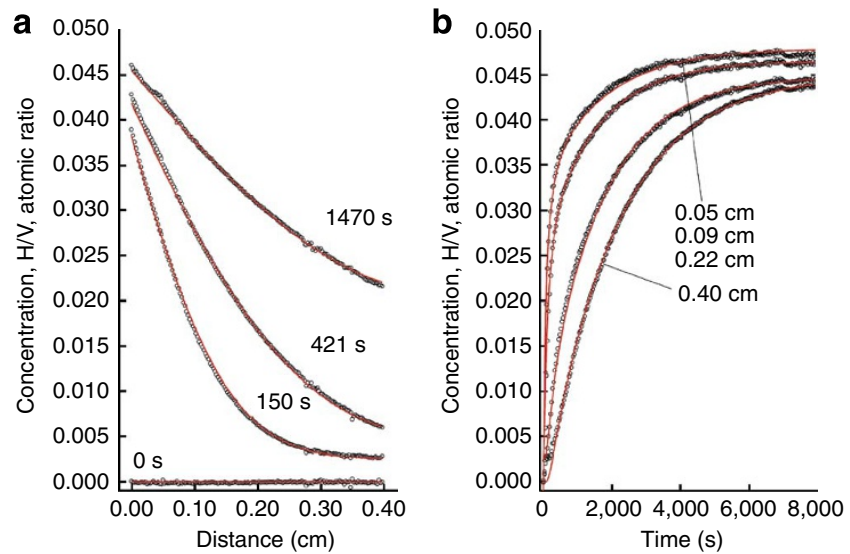

Figure 4 | Hydrogen concentration profiles at different times and positions. The concentration of hydrogen is plotted as the ratio of hydrogen to metal atoms over (a) distance and (b) time. Data is shown for different times and distances from the catalytic layer. The red lines represent least squares fits to the data using equation (8), as described in the Methods. Notice the sensitivity to the low amount of hydrogen with this method, where concentrations below 0.001 in $\mathrm{H} / \mathrm{V}$ can easily be distinguished

concentration as a function of position and time, using the results from the fitting.

The maximum equilibrium concentration in the sample is chosen by the temperature and the applied hydrogen pressure. This was used to perform diffusion measurements, at different temperatures, in the concentration range $0.01-0.50 \mathrm{H} / \mathrm{V}$. Measurements of the lateral diffusion at concentrations of $0.5 \mathrm{H} / \mathrm{V}$ are shown in Supplementary Movie 1. Given a film thickness of $50 \mathrm{~nm}$ or 330 monolayers of vanadium, a hydrogen concentration of $0.01 \mathrm{H} / \mathrm{V}$ corresponds to 3 effective monolayers of hydrogen. This sensitivity can be viewed as extraordinary and is unrivalled by any other measurement technique that we are aware of. We also notice large differences in the determined diffusion constants obtained at different hydrogen concentrations.

To obtain a more quantitative comparison between the results and the fitting, we display the evolution of concentration as a function of both time and position in Fig. 4. The evolution of the hydrogen concentration at different times is displayed in Fig. 4a, whereas the evolution at representative positions is illustrated in Fig. $4 \mathrm{~b}$. Besides the clearly identified lateral diffusion, a homogeneous increase in the concentration is clearly observed. This contribution was included in the simulations and amounts to about $1 / 20$ th of the total concentration.

\section{Discussion}

The diffusion rates of hydrogen in metals are comparable to the mobility of liquid water molecules and many aspects of hydrogen in metals can be understood by viewing hydrogen as a lattice gas or as a lattice liquid ${ }^{13,14}$. Diffusion in randomly oriented crystals should yield a weighted average of the rates in the principal crystallographic directions, ignoring the effect of grain boundaries on the diffusion rate. Depending on the influence of the grain boundaries and other imperfections in the material, the obtained diffusion rates could be significantly affected. The origin of the observed homogeneous increase in the concentration has not been identified. Discontinuous coverage of the protecting oxide layer is a likely source, while we cannot exclude other possible contributions. The presence of two components does not affect the results of our analysis, as we can clearly separate the contribution from both channels. We also notice that, in the absence of the lateral information on the 
concentration, this separation would not be possible, which would affect the determined diffusion coefficients.

Because of the discrete nature of the interstitial sites, the determined diffusion rate in different crystallographic directions will depend on the diffusion path in a single crystal. For example, in vanadium, the shortest distance between interstitial tetrahedral sites are the [110], [101] and [011] directions. These paths are the dominating directions of hydrogen transport in vanadium single crystals at low hydrogen concentrations. When calculating the relation between the diffusion- and the jump-rate, one needs to include these geometrical conditions. The results of the determined diffusion rates are therefore favourably compared with experiments using same boundary conditions. For example, a diffusion rate of $9(2) \times 10^{-5} \mathrm{~cm}^{2} \mathrm{~s}^{-1}$ for $c=0.02 \mathrm{H} / \mathrm{V}$ was obtained with [110] as the dominating diffusion direction $^{12}$, as compared with $D=8(1) \times 10^{-5} \mathrm{~cm}^{2} \mathrm{~s}^{-1}$ for $c=0.045$ $\mathrm{H} / \mathrm{V}$ obtained here. We notice an excellent agreement between the results from the two vastly different techniques.

The approach described here opens up new possibilities to address new fundamental aspects of hydrogen diffusion, such as exploration of the directional and concentration dependence, the influence of strain, as well as imperfections on the chemical diffusion of hydrogen. Most transition metals exhibit a similar overall change in the electronic structure on hydrogen absorption (for example, ref. 15). It is therefore reasonable to conclude that this approach can be used for analysis of diffusion rates of hydrogen in a wide range of metals. The lower limit in thickness of the samples is in the monolayer range, whereas the upper limit is determined by the penetration depth of light. This approach is therefore highly suited for investigations of hydrogen in thin film structures.

The use of this approach is not limited to determining the basic properties of hydrogen diffusion in materials. For example, metals with hydrogen as an added element often exhibit new and peculiar properties such as metal insulator transitions ${ }^{5}$, changes in magnetic properties ${ }^{3,16,17}$, changes in hardness and durability ${ }^{18-20}$, and modifications to superconducting properties ${ }^{3,21}$, to name a few. The possibility to determine the amount of hydrogen as a function of both position and time therefore opens up completely new avenues for combined measurements of concentration and different physical properties in thin films and multilayered structures. For example, the change in transmission can be used to determine the hydrogen concentration, whereas the rotation of polarization of the reflected beam can be used to determine the magnetization. In addition, the technique we describe opens up possibilities to investigate hydrogen migration as a function of strain, confinement and finite-size, all topics of importance for understanding the dynamics of hydrogen in confined spaces, such as nanomaterials.

\section{Methods}

Diffusion equation. A gradient in the chemical potential $\mu(x, t)$ (in one dimension) results in a current $s(x, t)$ (ref. 22)

$$
s(x, t)=-B\{c(x, t)\} c(x, t) \frac{\partial \mu(x, t)}{\partial x},
$$

where $B(c)$ is the (concentration dependent) mobility and $c$ is the concentration in atomic ratio $\mathrm{H} / \mathrm{V}$. Using the continuity relation (conservation of particles)

$$
\frac{\partial c(x, t)}{\partial t}+\frac{\partial s(x, t)}{\partial x}=0
$$

Fick's second law is obtained as

$$
\begin{gathered}
\frac{\partial c(x, t)}{\partial t}=\frac{\partial}{\partial x}\left(D(c) \frac{\partial c(x, t)}{\partial x}\right) \\
D(c)=B(c) c \frac{\partial \mu}{\partial c}
\end{gathered}
$$

For a non-interacting lattice gas, $\partial \mu / \partial c=k_{\mathrm{B}} T / c$ where $k_{\mathrm{B}}$ is the Boltzmann constant and $T$ is the absolute temperature, the Einstein relation can be written as: ${ }^{2,22}$

$$
D=B k_{\mathrm{B}} T \text {. }
$$

Because we are working with concentrations below $0.05 \mathrm{H} / \mathrm{V}$ and $T=493 \mathrm{~K}$ far above any phase boundaries, we argue that the hydrogen behave essentially as non-interacting particles and this justifies dropping the $c$ dependency of $D$ and $B$. However, for experiments at higher concentrations, the so-called thermodynamic factor, $f_{\text {therm }}=\left(c / k_{\mathrm{B}} T\right)(\partial \mu / \partial c)$ has to be evaluated to determine the diffusivity. Finally, the common form of Fick's second law is obtained:

$$
\frac{\partial c(x, t)}{\partial t}=D \frac{\partial^{2} c(x, t)}{\partial x^{2}}
$$

The boundary and initial conditions for the sample geometry shown in Fig. 1 is

$$
\begin{aligned}
& c(x, 0)=0 \forall x \geq 0, \\
& c(0, t)=c_{0} \forall t \geq 0, \\
& \left.\frac{\partial c(x, t)}{\partial x}\right|_{x=L}=0 \forall t \geq 0,
\end{aligned}
$$

where $L$ is the distance from the end of the active area. These conditions imply the sample being free of hydrogen before the measurement starts, no hydrogen can flow through the end of the sample and a constant hydrogen supply is available at the other end of the sample, where the catalytically active palladium is facing the hydrogen gas. The solution of equation (6) with the boundary and initial conditions in equation (7) is given by refs 23,24

$$
\begin{aligned}
c(x, t)= & c_{0} \sum_{k=1}^{\infty}\left[\operatorname{erfc}\left(\frac{2(k-1) L+x}{2 \sqrt{D t}}\right)+\right. \\
& \left.+\operatorname{erfc}\left(\frac{2 k L-x}{2 \sqrt{D t}}\right)\right](-1)^{k-1},
\end{aligned}
$$

where erfc is the complementary error function. The solution depends only on three parameters, $c_{0}, L$ and $D$, the first two of which can be measured independently. Offsets in time and position were also added as fitting parameters to reduce the impact on artificial effects on the fits. The series converges fast and only $k \leq 10$ need to be considered when comparing to the data. Representative solutions to the differential equation are shown in Fig. 4 along with experimental results.

Film growth. The $50 \mathrm{~nm}$ vanadium film was grown epitaxially on a double-sided polished $\mathrm{MgO}(001) 10 \mathrm{~mm} \times 10 \mathrm{~mm} \times 0.5 \mathrm{~mm}$ substrate using ultrahigh vacuumbased magnetron sputtering. The target purity was $99.98 \%$ and the base pressure in the system was $2 \times 10^{-7} \mathrm{~Pa}$ before deposition with the dominating residual gas being hydrogen. The sample was deposited from a vanadium target onto the substrate at $723 \mathrm{~K}$ on the heater. Argon, with purity better than $99.9999 \%$ at a pressure of $0.27 \mathrm{~Pa}$ was used as the sputtering gas. The sample was capped with a 5 -nm palladium layer. The palladium was sputtered from a target with $99.99 \%$ purity after cooling the sample to room temperature. $10 \mathrm{~nm}$ amorphous $\mathrm{Al}_{2} \mathrm{O}_{3}$ was subsequently deposited at $310 \mathrm{~K}$ with a piece of a microscope slide acting as a mask at one end of the sample. More detailed description of the growth and structural analysis can be found in ref. 11 .

Concentration calibration. The relation between hydrogen concentration and electronic structure is complex ${ }^{25}$, so we have resorted to a phenomenological approach of the conversion of optical transmission to hydrogen concentration, as described by Prinz et al. ${ }^{9}$ : the optical transmission was determined at different hydrogen concentrations using an optical spectrometer. From these measurements, the optical response was established as a function of wavelength and concentration. A wavelength where the square root of pressure (Sievert's law) was proportional to the logarithm of the detected transmitted intensity was thereby identified $(\lambda=639 \mathrm{~nm})$. Simultaneous measurements of the change in electrical resistivity were used to determine the change in concentration ${ }^{26}$. Verification of the concentration determination was obtained by comparing the results to previously determined isotherms, obtained from the hydrogen uptake in $50 \mathrm{~nm}$ thick vanadium films ${ }^{9,26,27}$.

\section{References}

1. Fick, A. On liquid diffusion. Philos. Mag. 10, 30-39 (1855)

2. Einstein, A. Über die von der molekularkinetischen Theorie der Wärme geforderte Bewegung von in ruhenden Flüssigkeiten suspendierten Teilchen. Ann. Phys. 17, 549-560 (1905).

3. Alefeld, G. \& Völkl, J. Hydrogen in Metals I (Springer-Verlag, Berlin, 1978). 
4. den Broeder, F. J. A. et al. Visualization of hydrogen migration in solids using switchable mirrors. Nature 394, 656-658 (1998).

5. Huiberts, J. N. et al. Yttrium and lanthanum hydride films with switchable optical properties. Nature 380, 231-234 (1996).

6. Remhof, A. et al. Switchable mirrors for visualization and control of hydrogen diffusion in transition metals. Phys. Rev. B 66, 020101 (2002).

7. Wang, Y., Pálsson, G. K., Raanaei, H. \& Hjörvarsson, B. The influence of amorphous $\mathrm{Al}_{2} \mathrm{O}_{3}$ coating on hydrogen uptake of materials. J. Alloys Compd. 464, L13-L16 (2008).

8. Alefeld, G. \& Völkl, J. Hydrogen in Metals II (Springer-Verlag, Berlin, 1978).

9. Prinz, J., Pálsson, G. K., Korelis, P. T. \& Hjörvarsson, B. Combined light and electron scattering for exploring hydrogen in thin metallic films. Appl. Phys. Lett. 97, 251910 (2010).

10. Fukai, Y., Kazama, S., Tanaka, K. \& Matsumoto, M. Hydrogen-induced states in $\mathrm{VH}_{x}$ and $\mathrm{VD}_{x}$ observed by soft $\mathrm{X}$-ray emission spectroscopy. Solid State Commun. 19, 507-509 (1976).

11. Isberg, P., Hjörvarsson, B., Wäppling, R., Svedberg, E. \& Hultman, L. Growth of epitaxial Fe/V (001) superlattice films. Vacuum 48, 483-489 (1997).

12. Völkl, J. \& Alefeld, G. The Gorsky effect: recent results. Il Nuovo Cimento B 33, 190-204 (1976).

13. Manchester, F. D. Lattice gas aspects of metal-hydrogen systems. J. LessCommon Met. 49, 1-12 (1976).

14. Wagner, H. \& Horner, H. Elastic interaction and the phase transition in coherent metal-hydrogen systems. Adv. Phys. 23, 587-637 (1974).

15. Smithson, H. et al. First-principles study of the stability and electronic structure of metal hydrides. Phys. Rev. B 66, 144107 (2002).

16. Leiner, V., Westerholt, K., Hjörvarsson, B. \& Zabel, H. Tunability of the interlayer exchange coupling. J. Phys. D 35, 2377-2383 (2002).

17. Kravtsov, E. et al. Proximity effect of vanadium on spin-density-wave magnetism in Cr films. Phys. Rev. B 70, 54425-54421 (2004).

18. Wipf, H. Hydrogen in Metals III (Springer-Verlag, Berlin, 1997).

19. Remhof, A. \& Borgschulte, A. Thin-Film Metal Hydrides. Chem. Phys. Chem. 9, 2440-2455 (2008)

20. Spitzig, W. A. \& Owen, C. V. Comparison of the effects of deuterium and of hydrogen on the low temperature strength and ductility of vanadium. J. LessCommon Met. 120, 301-305 (1986).

21. Manchester, F. D., San-Martin, A. \& Pitre, J. The H-Pd (hydrogen-palladium) System. J. Phase Equil. 15, 62-83 (1994).

22. Alefeld, G., Völkl, J. \& Schaumann, G. Elastic diffusion relaxation. Phys. Status Solidi 37, 337-351 (1970).
23. Crank, J. The Mathematics of Diffusion (Oxford University Press, London, 1970).

24. Podgorsek, R. P. \& Franke, H. Optical determination of molecule diffusion coefficients in polymer films. Appl. Phys. Lett. 73, 2887-2889 (1998).

25. Andersson, P. H., Fast, L., Nordström, L., Johansson, B. \& Eriksson, O. Theoretical study of structural and electronic properties of $\mathrm{VH}_{x}$. Phys. Rev. $B$ 58, 5230-5235 (1998).

26. Bloch, J., Pejova, B., Jacob, J. \& Hjörvarsson, B. Hydrogen-vanadium system in thin films: Effect of film thickness. Phys. Rev. B 82, 245428 (2010).

27. Bloch, J., Hjörvarsson, B., Olsson, S. \& Brukas, R. Reversible structural change and thermodynamic properties of hydrogen in thin vanadium films. Phys. Rev. $B$ 75, 165418 (2007).

\section{Acknowledgements}

Financial support from the Swedish Research Council and the Knut and Alice Wallenberg Foundation, is gratefully acknowledged.

\section{Author contributions}

G.K.P. and B.H. developed the idea behind the measurements. The experimental set-up was designed by A.B., G.K.P. and B.H., and measurements were performed by A.B. The analyses were made by G.K.P. and A.B., and the manuscript was written by G.K.P., B.H. and A.B., with input from all the authors. The sample was grown by A.Z. and G.K.P., who also made the structural analysis. M.W. provided input on the results and the analysis. G.K.P., A.B. and B.H. made the figures.

\section{Additional information}

Supplementary Information accompanies this paper at http://www.nature.com/ naturecommunications.

Competing financial interests: The authors declare no competing financial interests.

Reprints and permission information is available online at http://npg.nature.com/ reprintsandpermissions/

How to cite this article: Pálsson, G.K. et al. Using light transmission to watch hydrogen diffuse. Nat. Commun. 3:892 doi: 10.1038/ncomms1897 (2012).

License: This work is licensed under a Creative Commons Attribution-NonCommercialNoDerivative Works 3.0 Unported License. To view a copy of this license, visit http:// creativecommons.org/licenses/by-nc-nd/3.0/ 